\title{
Pensamiento arbitrista y estructuras institucionales en la carrera de Indias (siglo XVII): entre la desincentivación y la represión*/
}

\author{
Arbitrist Thought and Institutional Structures regarding \\ the Spanish Treasure Fleets (17th century): \\ Between Disincentivization and Repression
}

José Manuel Díaz Blanco

Universidad de Huelva

El arbitrismo castellano del siglo XVII mostró una gran preocupación por el comercio colonial y, sin embargo, la carrera de Indias participó en la cultura arbitrista de forma moderada. ¿Cómo puede explicarse esta paradoja? Este artículo ofrece como respuesta una argumentación basada en tres ideas: 1) existió una relación contradictoria entre la cultura arbitrista y el aparato institucional de la carrera; 2) esa relación varió a lo largo del siglo XVII: durante las primeras décadas las instituciones desincentivaron el arbitrismo y, durante las últimas, tendieron más a reprimirlo; 3) tal evolución se debe en gran medida al desplazamiento del centro de la Carrera desde Sevilla hasta Cádiz.

PAlabras ClaVE: Arbitrismo; Carrera de Indias; Siglo XVII; Horacio Levanto; Juan Cano.

The Castilian arbitrism of the 17th century demonstrated great concern about the colonial trade; however, the Spanish Treasure Fleets are only moderately present in arbitrista culture. How can this paradox be explained? This paper proposes an argument based on three ideas: 1) there was a contradictory relationship between the arbitrista culture and the institutional system of the Fleets; 2) this relationship changed throughout the 17th century: during the first decades of the century the institutions disincentivized arbitrismo and, during the last ones, they tended to repress it; 3) this evolution is largely due to the transfer of the Fleets from Seville to Cadiz.

KEYwords: Arbitrism; Spanish Treasure Fleets; 17th Century; Horacio Levanto; Juan Cano.

* Este trabajo se ha realizado con un contrato del programa Juan de la Cierva del Ministerio de Economía y Competitividad de España, expediente JCI-2011-11153, vinculado al proyecto de investigación I+D+i «Extranjeros y pueblos indígenas en la mentalidad hispana del siglo XVIII: estrategias represivas y procesos de integración en España y América» (referencia: HAR2010-15141), financiado por el Ministerio de Economía y Competitividad de España y por FEDER de la Unión Europea. Agradezco a Arrigo Amadori la gentil invitación a participar en este dossier y a Manuel Herrero Sánchez sus expertas indicaciones sobre la materia. 
El arbitrismo fue una de las corrientes culturales más características de la España del siglo XVII. ${ }^{1}$ Las razones de su proliferación son sobradamente conocidas; abundaron en aquella centuria diversas modalidades de percepción de una crisis frente a la cual muchos hombres reaccionaron, intentando hacer llegar al gobierno y a la sociedad ideas que pudiesen contribuir a mejorar la situación. Los arbitrios de los arbitristas eran recetas contra los supuestos males de un tiempo difícil para la monarquía en general y para Castilla en particular. ${ }^{2}$ Algunos tuvieron su origen en reflexiones poco sólidas, que merecían con frecuencia el menosprecio de los contemporáneos; $;^{3}$ en cambio, otros eran tratados serios, redactados por auténticos patriotas que ponían sus cualificados esfuerzos al servicio de la comunidad y de la corona. ${ }^{4}$

En los textos arbitristas se percibe una preocupación significativa por América, especialmente respecto de la plata que enviaba a Europa y del comercio que unía ambos continentes a través de la estructura monopolística centralizada en el puerto de Sevilla. El hecho es normal, si se tiene presente que todos estos factores fueron elementos esenciales de la economía castellana de la época. Sin embargo, producen cierta sorpresa o curiosidad algunos aspectos que salen a la luz en cuanto se analiza la relación entre el arbitrismo y la carrera de Indias. En primer lugar, los grandes arbitristas que se preocuparon por la economía atlántica no estaban directamente relacionados con ella; ninguno de ellos fue uno de los mercaderes u oficiales reales que vivieron la carrera desde dentro. Y, más significativamente aún, casi ninguno de estos últimos formó parte del movimiento arbitrista. La carrera no generó ningún arbitrista de relieve a lo largo del siglo XVII, salvo raras excepciones entre las que merece la pena destacarse al mercader

1 La historiografía reconoce en el XVII el siglo de mayor auge del arbitrismo, pero no circunscribe el fenómeno solo a este período de tiempo. El primer gran arbitrista fue Luis Ortiz y su Memorial de 1558. Dubet, 2003, demuestra que el proyecto de los erarios de Valle de la Cerda tenía sus orígenes en otros autores del siglo XVI y que el mismo Valle de la Cerda ya lo había defendido en tiempos de Felipe II. Almarza, 1990, vincula arbitrismo con siglo XVIII.

2 El arbitrismo se asocia particularmente a Castilla, pero no solo floreció allí, como demuestran los estudios sobre la expansión del fenómeno en Aragón (Perdices de Blas y Sánchez Molledo, 2007), América (Falcón Ramírez, 1988) y otros territorios europeos de la monarquía, como Flandes o Nápoles (véanse las contribuciones de Miguel Ángel Echevarría Bacigalupe y Gaetano Sabatini, en Sabatini y Dubet, 2008).

3 Vilar, 1973.

4 Visiones de conjunto sobre el pensamiento arbitrista y lecturas revalorizadoras del fenómeno en Colmeiro, 2005 [1861]; Larraz, 1963 [1943]; Sureda Carrión, 1949; Vilar, 1962; Correa Calderón, 1981; Elliott, 1982 y 1990; Gutiérrez Nieto, 1986; Perdices de Blas, 1996; Perdices de BlasReeder, 1998; Feros, 2002; Dubet, 2003; García Guerra, 2003; Alloza Aparicio, 2009, entre otros. 
borgoñón Juan Cano, ya en el reinado de Carlos II, que en realidad tuvo una dimensión intelectual mucho menor de la que podría haber alcanzado, a causa de circunstancias que se explicarán más adelante. ${ }^{5}$

¿A qué se debe esta aparente paradoja? El texto que se ofrece a continuación tiene como objetivo responder a esta pregunta. Una propuesta sencilla podría conformarse con afirmar que no existió una relación directa entre el arbitrismo y la carrera de Indias porque esta pudo no haber experimentado ningún tipo de crisis a lo largo del siglo XVII. Algunas propuestas revisionistas descartan taxativamente la existencia de la recesión que antes se daba por asumida. ${ }^{6}$ Sin embargo, el arbitrismo no nacía directamente de la crisis en sí, sobre cuya existencia puede debatir la historiografía, sino de la percepción de los contemporáneos de que esa crisis existía. $\mathrm{Y}$ es imposible negar que dicha percepción, equivocada o no, estuvo muy extendida. Existe una infinitud de testimonios al respecto. Como el del consulado, que resumió con precisión ese estado de opinión en 1637, cuando lamentó, como tantas otras veces, «la estrecheza de los tiempos y [...] hallarse los caudales de todas las personas de que este comercio se compone totalmente acabados». ${ }^{7}$ Afirmaciones de este tipo recorren todo el siglo XVII andaluz. ${ }^{8}$ Por tanto, el interrogante parece requerir otra solución.

La hipótesis que aquí se propone relaciona la escasez del arbitrismo con el alto grado de institucionalización interna de la carrera de Indias. Es decir, quiere ofrecerse como idea central la existencia de una relación contradictoria entre la cultura arbitrista y las estructuras institucionales de la carrera de Indias. Este planteamiento se vincula con los estudios que han analizado las relaciones entre el proyectismo español y el poder político. ¿Cómo debe estudiarse el arbitrismo? Desde la óptica de la historia del pensamiento, tal vez puede resultar satisfactorio un análisis que desgrane hasta el detalle el contenido de los textos. Pero, para la historia general,

5 Además de la mencionada diferencia cuantitativa en el nivel de producción escrita, las diferencias cualitativas entre unos y otros autores pueden deducirse con facilidad. En este artículo, el arbitrista es siempre aquel que ofrece arbitrios al poder político para solucionar una situación de crisis. Sin embargo, había quien podía hablar desde una experiencia directa de la materia, con un conocimiento profesional y especializado de la misma, y otros que la abordaban desde ámbitos totalmente ajenos a ella, desde un posicionamiento puramente teórico, aunque también legítimo. Lógicamente, el conocimiento sobre la carrera de Indias de unos y otros no podía ser el mismo.

6 Oliva Melgar, 2004 y 2005.

7 Archivo General de Indias (AGI), Consulados, 3, 3. ${ }^{a}$ junta en que se concede por el comercio el servicio y ofrecimiento tocante a el uno por ciento de los infantes, Sevilla, 17 de febrero de 1637.

8 Díaz Blanco, 2012a. 
posee una relevancia mayor conocer las conexiones entre la conciencia crítica y el poder político. Posiblemente, el ejemplo más conocido de este enfoque se encuentra en la biografía del conde duque de Olivares de John $\mathrm{H}$. Elliott, donde se retrata el régimen del valido como el beneficiario de una «herencia reformista» que puso en práctica hasta que entró en contradicción con las resistencias sociales y el desarrollo de una política reputacionista. ${ }^{9}$ Pero hay muchos otros estudios que indagan en el binomio pensamiento arbitrista-poder político, como los de Antonio Feros Carrasco, Elena M. García Guerra, Ángel Alloza Aparicio o, fuera de un arbitrismo estrictamente económico, Juan Hernández Franco, ${ }^{10}$ entre otros. Tal vez, el enfoque más consciente o explícito metodológicamente sea el empleado por Anne Dubet, que no definió su obra sobre los arbitrios de Luis Valle de la Cerda como un análisis textual, sino como un libro donde se «estudia la negociación política que se desarrolla entre la corona y los poderes concurrentes [...] en un marco preciso: los sucesivos intentos de reforma del crédito público y privado inspirados en el proyecto [...] promovido por Luis Valle de la Cerda». ${ }^{11}$ Ha afirmado respecto a los arbitristas: «savoir comment leurs textes ont été lus et utilisés — qu'on les ait compris ou nonest aussi important que de saisir leur signification». ${ }^{12}$

El fenómeno que pretende retratarse aquí fue evolucionando a lo largo del siglo XVII. En efecto, una visión estática de esta difícil relación entre la cultura arbitrista y las instituciones de la carrera no tiene visos de ser acertada. Pueden distinguirse, al menos, dos fases dentro de su proceso de desarrollo, que deben ponerse en relación con el conocido proceso de sustitución entre Sevilla y Cádiz en la capitalidad de la carrera. Durante la primera etapa, en la que Sevilla todavía ostentaba una primacía indiscutible, la estructura institucional actuó como un elemento desincentivador del arbitrismo; sin embargo, durante el segundo período, cuando Cádiz comenzó a desarrollarse como alternativa de Sevilla, la estructura institucional se convirtió en algo más: un instrumento represor del pensamiento arbitrista. La contradicción se había agravado conforme avanzaba el siglo y la comunidad mercantil iba concentrándose en Cádiz. Establecer una cronología precisa para este proceso de cambio es tan difícil y anti-histórico como pro-

9 Elliott, 1990

10 Feros Carrasco, 2002; García Guerra, 2003; Alloza Aparicio, 2009; Hernández Franco, 2011.

11 Dubet, 2003, 21.

12 Dubet, 2000. 
poner fechas definidas para el reequilibrio de los puertos andaluces dentro del comercio colonial español. Pero la cesura podría situarse en torno a 1650 о 1660 .

\section{La fase sevillana: desincentivación}

El momento arbitrista por excelencia es el reinado de Felipe III, los inicios del siglo XVII. Esta afirmación, que no es nueva, ha podido demostrarse desde una perspectiva cuantitativa. Basándose en el admirable repertorio de arbitristas construido por Evaristo Correa Calderón, ${ }^{13}$ y aun asumiendo que podría perfeccionarse, Gaetano Sabatini y Anne Dubet han establecido una cronología que alcanza su punto cenital en los reinados de Felipe III y Felipe IV. ${ }^{14}$ El enfoque cualitativo termina de afinar el análisis, aunque posea un componente innegablemente subjetivo: además de la abundancia de arbitristas, a comienzos del siglo XVII escribieron los más conocidos e influyentes de ellos: Martín González de Cellorigo, Baltasar Álamos de Barrientos, Luis Valle de la Cerda, Sancho de Moncada... El reinado de Felipe III es la edad dorada del arbitrismo en Castilla. ${ }^{15}$ Incluso el surgimiento literario de la palabra, presente en el Tesoro de Covarrubias (1611), acontece entonces en el Coloquio de los perros cervantino (1613). ${ }^{16}$

Los grandes arbitristas de Castilla dedicaron importantes reflexiones a América y lo hicieron desde una perspectiva crítica. ${ }^{17}$ Los territorios indianos habían actuado como mercado y como suministradores de metales preciosos, a pesar de lo cual los arbitristas denunciaron que se había convertido en un elemento de empobrecimiento para España. La paradoja se explica por la utilización que los castellanos habían hecho de las riquezas de América. En las obras de Martín González de Cellorigo, Sancho de Moncada, Pedro Fernández de Navarrete o Francisco Martínez de Mata se deploraba cómo los españoles habían sucumbido ante la ilusión de la rique-

13 Correa Calderón, 1981.

14 Sabatini y Dubet, $2008,869$.

15 Dubet, 2000: «Parmi les [arbitristes] plus célèbres: Cristóbal Pérez de Herrera, Sancho de Moncada, Martin González de Cellorigo ou encore Luis Valle de la Cerda». Con Felipe III, también se observa un florecimiento dentro del arbitrismo americano: Díaz Blanco, 2009a y 2012b; Herzog, 2008, 930-931, vincula el arbitrismo americano con el reinado de Felipe III a través de la figura de Felipe Guamán Poma de Ayala.

16 Vilar, 1963, 48.

17 Elliott, 1990; Perdices de Blas, 1996, 39-45. 
za fácil, provocada por las riadas de metales preciosos llegadas del océano, y se habían entregado al vicio de la ociosidad, mientras los extranjeros se adueñaban de la estructura económica nacional en beneficio propio y en detrimento de España. ${ }^{18}$ La cultura arbitrista sistematizó así una crítica contra la carrera de Indias, que denunciaba el escaso grado de riqueza que inyectaba en la economía del país. ${ }^{19}$ Sin embargo, quienes lo afirmaban no eran cargadores. Cellorigo era un abogado vallisoletano; Moncada, un teólogo toledano; Martínez de Mata, un franciscano motrileño que vivió en Sevilla, pero sin guardar relaciones personales significativas con la élite comercial hispalense..$^{20}$ Jamás se habían visto involucrados en la dura realidad de los negocios coloniales ni vivieron su desarrollo cotidiano. La mayoría ni siquiera conocía a sus principales protagonistas. Hablaban sin conocimiento directo, aunque no por ello sus opiniones dejan de tener un elevado interés.

Mientras Cellorigo, Moncada y otros escribían sobre el comercio colonial, la fase sevillana de la carrera de Indias daba sus últimas bocanadas. La ciudad del Guadalquivir cobijaba entonces una nutrida comunidad de mercaderes que vivía azorada por una enorme cantidad de problemas, similares o coincidentes con los que denunciaban los arbitristas. Sin embargo, debe esforzarse mucho el historiador para encontrar algún arbitrista entre ellos. Desde luego, ninguno alcanzó el nivel de los mejores representantes del género y muy pocos de ellos lo cultivaron siquiera en un tono menor. Entonces, ¿qué ocurrió con las tribulaciones de las grandes casas mayoristas? ¿Qué fue de sus quejas, de sus problemas y de las soluciones que proyectaban para darles fin? ¿Se las llevaron el viento y el tiempo, fuera del resguardo que podía proporcionarles el papel escrito? No, en absoluto. Hay una enorme masa de papeles que ha retratado las cuitas de los mercaderes, tan voluminosa que posiblemente desborda la capacidad de lectura de un solo hombre. Pero no está escrita directamente por ellos, sino por las instituciones que gobernaban la carrera de Indias.

La estructura institucional de la carrera daba cabida a un conjunto de organismos públicos desigualmente conocidos por la investigación histórica. El Consejo de Indias, que fue el más elevado jerárquicamente, ha sido

18 González de Cellorigo, 1991 [1600]; Moncada, 1974 [1619]; Fernández de Navarrete 1982 [1626]; Martínez de Mata, 1971.

19 Herzog, 2008, 926.

20 Antes al contrario, era un defensor de los pobres y contó con la enemistad de algunos aristócratas conspicuos como el caballero veinticuatro Martín de Ulloa. 
objeto de un nutrido conjunto de estudios y su historia se halla razonablemente bien construida; ${ }^{21}$ la Casa de la Contratación ha recibido una atención menor y aún se encuentra huérfana de una monografía que le haga verdadera justicia: ${ }^{22}$ el Consulado de Cargadores a Indias es prácticamente un desconocido del que, por increíble que parezca, apenas se manejan los aspectos más esenciales. ${ }^{23}$ Sin duda, otras instituciones influyeron sobre la vida pública de la carrera, dentro de la administración central y del marco municipal de las ciudades andaluzas, pero estas tres fueron las más relevantes junto con el propio monarca a lo largo del siglo XVII: ${ }^{24}$

- El Consejo de Indias nació como tal en 1524, dentro del conjunto del sistema polisinodial cortesano que ayudaba a los reyes en el gobierno de la monarquía. Fue la máxima autoridad en el gobierno colonial, jurisdicción que incluía la estructura comercial de la Baja Andalucía. En el desempeño de sus funciones, el Consejo habló con frecuencia a favor de los cargadores, comprendió sus problemas y, por lo general, defendió sus intereses en Madrid.

- La Casa de la Contratación se creó en 1503 y fue el primer organismo dedicado específicamente a gestionar la relación de España con los territorios americanos. No tuvo una gran capacidad de decisión; fue una instancia fundamentalmente ejecutora de las líneas de actuación diseñadas en Madrid. Su misión era representar a la monarquía en Sevilla, aunque terminó identificándose en gran medida con los mercaderes y la sociedad local.

- El Consulado de Cargadores surgió en 1543. A través del triunvirato formado por el prior y los dos cónsules, la comunidad de mercaderes podía debatir sobre el estado de carrera de Indias, definir sus aspiraciones y defenderlas frente a quien correspondiera.

La intensidad del diálogo institucional que sostuvieron estos organismos no es nada que necesite demostrarse. Está documentada la vivacidad del debate político entre el gobierno de Madrid y la burguesía sevillana a

21 Schäfer, 2003 [1935]; García Pérez, 1998.

22 Lo denuncian, entre otros, Crespo Solana, 1996, 11; Bernal, 2003; Andújar Castillo, 2008, 162. El quinto centenario de su fundación generó una gran obra miscelánea: Vila, Acosta y González, 2003, pero sigue siendo necesaria la visión de conjunto que ofrece Schäfer, 2003 [1935].

23 Vila Vilar, 1999.

24 Otras visiones de conjunto de este organigrama de gobierno, cada una con sus matices, en García-Baquero, 1992 y Bernal, 2005, 178-187. 
través de ellos. ${ }^{25}$ Estas conclusiones pueden reforzarse desde una perspectiva cuantitativa a través del análisis de los fondos de correspondencia que conserva actualmente el Archivo de Indias en las (sub)secciones Indiferente General, Contratación y Consulados. La correspondencia cruzada entre las tres instituciones ocupa cientos de legajos y libros. ${ }^{26} \mathrm{La}$ abundancia de estos fondos queda patente y se encuentra lejos de haber sido agotada por la investigación histórica. Antes al contrario, algunas series, especialmente la correspondencia del consulado, se hallan prácticamente vírgenes. Dentro de sus volúmenes aguardan millones de documentos cruzados entre el Consejo, la Casa y el Consulado, que son la evidencia más directa de la comunicación sistemática entre ellas. A través de ese diálogo se afrontaban los problemas y los retos de la carrera de Indias en el siglo XVII, que no fueron pequeños.

En ese contexto político, la prueba negativa, la inexistencia de una corriente poderosa de arbitrismo comercial puro, parece concluyente. Puesto que, sin duda, nada hubiera impedido a los mercaderes expresarse al modo arbitrista; de hecho, algún caso existe y se estudiará a continuación. Pero la realidad que se impuso fue actuar normalmente a través de las instituciones oficiales. La vida política de la carrera de Indias se desarrolló dentro de un modelo institucionalizado, no de un modelo individualista. El arbitrismo, el proyectismo, los deseos de personas concretas por cambiar la realidad y mejorarla, no escaparon a este estado de cosas. Si nuevos descubrimientos documentales no modifican esta visión de base, puede concluirse que el entramado institucional no fomentó el arbitrismo dentro de la carrera, aunque podría haberlo hecho, sino que lo desincentivó.

Resulta necesario aclarar que la premisa de esta afirmación no es la de una perfecta sintonía entre la comunidad mercantil y el marco institucional, mucho menos si se establece otra condición previa: la homogeneidad de ambos planos. La conclusión no necesita esa premisa ni de hecho la incluye, porque no es cierta. El mundo de la carrera de Indias era enormemente complejo y conflictivo, la comunidad mercantil no era homogénea y el marco institucional tampoco. Antes al contrario, el elemento social y el entramado institucional se encontraban fuertemente fragmentados y esa fragmentación era la que permitía consolidar el entendimiento entre ambos.

25 Díaz Blanco, 2012a.

26 AGI, Contratación, 5.016-5.063, 5.091, 5.093, 5.113-5.142, 5.170-5.184 y 5.197-5.214. AGI, Consulados, 49-60 y 107-154. AGI, Indiferente General (en adelante IG), 1.116-1.201. Véase Tariego, 1801; Heredia Herrera, 1979, 31-34; Archivo General de Indias, s.f. 
Los diversos colectivos sociales solían encontrar una institución que representase sus intereses frente a otros colectivos, a quienes también defendía otra institución enemistada con la anterior. Los agentes establecían así bloques de alianzas para fortalecer su posición en los conflictos en los que se veían frecuentemente implicados. ${ }^{27}$

$$
* * *
$$

Uno de los pocos casos en los que puede detectarse cierta actividad como arbitrista vinculada a un cargador a Indias lleva a la vida de Horacio Levanto. Levanto fue el pilar sobre el que se sustentaron los miembros de una dinastía que, en palabras de D. Antonio Domínguez Ortiz, «reunieron en Andalucía uno de los capitales más grandes de la época». ${ }^{28}$ Los datos que se conocen sobre Levanto evidencian una vida muy intensa y fructífera. Nació en Génova, pero al igual que muchos ligures desarrolló su actividad profesional en el mundo hispánico. Desde 1594, aproximadamente, residió durante varios años en América, en Puebla de los Ángeles, donde se avecindó, se compuso con la monarquía y conoció de primera mano aquel lejano mundo colonial, en el que se consumían los productos cargados en las flotas de Indias. En 1610 consiguió una carta de naturaleza ${ }^{29}$ y se estableció en Sevilla, haciendo gala de una envidiable posición económica. ${ }^{30}$ Eludió sin dificultad las presiones a las que el consulado sometió a los empresarios extranjeros ${ }^{31}$ y no dejó de incrementar su fortuna. Se le han documentado tratos importantes con Nueva España, ${ }^{32}$ hizo negocios de todo tipo y ocupó importantes cargos institucionales, mediando negociación económica previa con la corona: fue medidor mayor de la alhóndiga de

27 Diversos ejemplos en diferentes coyunturas estudiados en Collado Villalta, 1983 y Díaz Blanco, 2009b y 2012a.

28 Domínguez Ortiz, 1996, 29-30.

29 AGI, México, 1, n. 400; consulta del Consejo de Indias, Madrid, 27 de noviembre de 1610, cuya resolución positiva dio como resultado AGI, Contratación 596b y AGI, IG, 449, Carta de naturaleza de Horacio Levanto, Madrid, 21 de diciembre de 1610.

30 AGI, IG, 596b. En efecto, no se citan bienes inmuebles, de los que seguramente carecería en propiedad por llevar poco tiempo residiendo en Sevilla. Presenta dinero líquido, deudas y mercancías en Andalucía, Génova, México y Filipinas. Por cierto, uno de los deudores sevillanos es un don Francisco Pacheco, que tal vez podría identificarse con el conocido pintor, suegro de Diego Velázquez.

31 Díaz Blanco, 2012a. fortuna».

32 García Fuentes, 1997, 205, 234 y 309, que resalta que Levanto «amasó una auténtica 
Sevilla, fundidor de la casa de la moneda de México o tesorero de la casas de la moneda de Sevilla y Granada. ${ }^{33}$

Levanto fue el autor de un Memorial sobre el trato de la China con Nueva España y estos reinos, impreso en una fecha comprendida entre 1620 y $1622 .{ }^{34}$ La obra no es comparable a los mejores ejemplares de la literatura arbitrista. Se trata de un opúsculo de menos que mediana extensión, pero conoció la imprenta (aunque fuera en una edición algo apresurada) ${ }^{35}$ y posee la suficiente entidad para reservarle un análisis individualizado. Parece discernirse en sus páginas la funcionalidad dentro de un proceso de decisiones institucionales. No lo revela solo la dedicatoria a don Fernando Carrillo, presidente del Consejo de Indias, que es el elemento político más evidente, sino también una curiosa estructura de redacción doble: el Memorial tuvo una forma inicial más breve, que no ha llegado directamente a la actualidad, a la cual Levanto le añadió otros párrafos que se localizan visualmente en el impreso por haberse destacado en cursiva. Estos añadidos parecen obedecer a distintas razones y se diría que una de ellas fue responder a dudas u objeciones planteadas a la versión inicial. El texto revela la existencia de un diálogo, que tal vez fue un diálogo institucional. Podría especularse con la posibilidad de que el trasfondo se encontrase en el tránsito entre los reinados de Felipe III y Felipe IV. ${ }^{36} \mathrm{Al}$ desaparecer un rey y sucederle otro nuevo, acompañado de nuevos consejeros y hombres de confianza, podían darse las circunstancias adecuadas para solicitar un viraje radical en una cuestión política que debió de preocupar a muchos mercaderes, especialmente a aquellos que hacían sus negocios preferentemente con México.

A comienzos del siglo XVII la percepción sobre los problemas del comercio atlántico comienzan a multiplicarse en Sevilla. Los afectados esgrimen diversas causas y una de ellas sorprende al lector actual por la amplia comprensión de los mercados internacionales que supone en los

33 Vinculado a su desempeño como tesorero de la casa granadina, surgió un impreso interesante: Biblioteca Universitaria de Sevilla, A 110/111(14), Por Oracio Leuanto Tesorero de la casa de la moneda desta ciudad. En el pleito con el Licenciado Manuel Ruyz Aguado, Alcalde de la dicha casa, y los Capataces della. Sobre la querella de que excede de su jurisdición, en conocer de vna demanda que los dichos Capataces han puesto al dicho Tesorero, Granada, Martín Fernández, c. 1625.

34 Biblioteca Nacional de España (BN), R/17.270. El texto no tiene fecha y los elementos que ayudan a proponer una datación no permiten, desgraciadamente, una mayor precisión.

35 Lo ejemplifica el título de la obra, que reza en realidad Memorial sorbe [sic] el trato de la China con Nueva España y estos reinos.

36 Una descripción clásica de este momento en Elliott, 1990, a las que se han añadido otras posteriormente, como las de Feros Carrasco, 2002. 
contemporáneos, pese a sus limitados medios de información: la competencia asiática en los mercados americanos. ${ }^{37}$ China introducía en las Indias, sobre todo en Nueva España, productos extremadamente competitivos gracias a sus bajos precios, acompañados de una calidad más que aceptable. Era imposible competir contra ellos en un mercado libre, por lo que se buscaba la intervención institucional. En Andalucía, pocas personas podían escribir con mayor autoridad sobre la materia que Horacio Levanto, que hablaba desde la experiencia de haber residido más de quince años en México. Según su testimonio, «grande es el trato, señor, que ay de la gran China a la ciudad de Manila, cabeça de la Isla de Luzon». Lo protagonizaban «cada año cantidad de Chinos, que por otro nombre llaman Sangleyes», comerciando con textiles de algodón y seda. El atractivo de las ropas chinas en Manila se debía en parte a que eran muy aptas para la reexportación a la Nueva España, donde encontraban un amplio mercado e incluso la plataforma donde seguir haciendo ruta hasta Europa. Tal éxito se fundamentaba en una combinación de calidad y baratura con la que los productos europeos parecían no poder competir. En México las mercancías chinas luchaban con éxito entre las élites criollas del país y se imponían entre los sectores de población indígena, entre los pobres, la mayor parte de la sociedad urbana colonial: «los dichos texidos de Algodón son tambien muy gastables, y particularmente entre los Indios, que no apetecen los que les van destos Reynos de lino, y cañamo». La baratura con una calidad razonable era la clave, acompañada por la inteligencia empresarial de los mercaderes chinos y la flexibilidad de su estructura productiva. ${ }^{38}$

Aunque el impacto asiático en América tenía su epicentro en México, también se estaba extendiendo a Perú. Si no se tomaban cartas en el asunto, el desafío a la legalidad vigente no dejaría de agravarse. ¿Qué hacer en estas circunstancias? Levanto recomendaba intervenir los mercados para beneficiar a la producción europea con dos medidas principales. En primer lugar, «Qve no se pudiera nauegar de la dicha Isla de Luzon a la Nueua España texido ninguno de seda, ni ropa hecha dellos, que es lo que haze el daño a los que se labran en estos Reynos». ${ }^{39}$ En segundo,

37 Respecto al espacio económico creado en el Pacífico, entre el Extremo Oriente y México, protagonizado por el célebre galeón de Manila, véanse, entre otros, Schurz, 1992 [1939], Alfonso Mola y Martínez Shaw, 2000; Martínez Shaw y Alfonso Mola, 2007; Bernabéu Albert y Martínez Shaw, 2013 y Bernabéu Albert, 2014.

38 Memorial, 1.

39 Ibidem, 2. 
mandar, que por cuenta de la real hazienda de su Magestad, se comprassen cada año en Manila mil caxones de seda madexa, pelos, y tramas [...]. Y como la experiencia ha mostrado, por no auer venido de dos años a esta parte, como solia, por via de Nueua España, ha hecho gran falta al trato della, del qual se sustenta mucha cantidad de personas, assi hombres, como mujeres. Y no solamente à resultado daño a esta ciudad de Seuilla, que tambien à tocado a Toledo, y a otras partes del Reyno [de Castilla], donde la solian lleuar. Y muchos texedores, y otros oficiales del dicho trato, por no tener que trabajar en sus tierras, se an ido a las Indias, y otras partes. ${ }^{40}$

Las directrices del pensamiento económico de Levanto son muy claras: moderación de las Indias como espacio de producción y consumo de manufacturas propias o de potencias extranjeras como China; potenciación de Filipinas como foco de suministro de materias primas, la seda en madeja; y apuntalamiento de Castilla como espacio de producción de los bienes elaborados que se consumían en Hispanoamérica y en el Extremo Oriente. Se trata de un pensamiento de claro perfil colonialista, que trata de dificultar la madurez económica de territorios políticamente sometidos para que ese control político siguiera sirviendo a una relación económica desigual, evidentemente favorable a la metrópoli española.

El Memorial de Levanto es apasionante y, pese a su brevedad, merecería un estudio monográfico que no es posible dedicarle aquí. A los efectos del presente trabajo, interesa considerarlo como un ejemplo de literatura arbitrista aplicado a una cuestión en la que la actividad institucional pura resultó igualmente funcional. O incluso más funcional. En efecto, en el marco de las instituciones sevillanas se discutió en no pocas ocasiones sobre los efectos de la producción asiática en los mercados novohispanos. En 1610, por ejemplo, los cargadores a Indias vinculados al consulado se sintieron oprimidos por un entorno económico adverso, de cuyas claves se discutió en una junta de comercio celebrada en la Casa Lonja. Se hallaba presente el gobierno consular - Miguel Polo, Antonio de Montalvo y Francisco de Mandojana — ${ }^{41}$, la plana completa de consiliarios —Diego Álvarez Gaibor o Rodrigo de León Garavito, entre ellos-y varios mercaderes de prestigio - Pedro de Avendaño Villela, Jorge de Reinoso y otros más- L Los galeones de Tierra Firme y la flota de Nueva España habían llegado a Sanlúcar de Barrameda y Cádiz, pero la esperada noticia se había amargado, acompañada de comentarios comprobados sobre el escaso éxito de los negocios que se habían efectuado. Según las propias palabras de los

40 Ibidem, 3.

41 Heredia Herrera, 1985. 
afectados, «es notorio las malas correspondencias y ventas que hubo en entrambas provincias de Nueva España y Tierra Firme». La razón principal: los mercados americanos estaban saturados y el exceso de oferta había generado precios de venta al público bajos con los que era imposible rentabilizar un negocio tan costoso como el comercio colonial.

Varias causas actuaban sobre la situación de los mercados, aunque todas estaban relacionadas con la competencia derivada de otros focos de oferta que actuaban desde diversas condiciones de legalidad. En Perú resultaban durísimos los efectos del intercambio comercial fraudulento que se realizaba desde la capital económica, Potosí, con Brasil y el Río de la Plata y desde allí con diversas naciones europeas que tenían vedado el acceso a los mercados americanos. No menores eran los perjuicios producidos en los dos virreinatos por los navíos negreros procedentes de Guinea, que traían de contrabando otro tipo de mercancías que introducían sin permisos y sin pagar derechos a la Administración. Pero

el mayor [daño] de todos es la saca de dos millones y más de plata que cada un año sale de la dicha provincia [de Nueva España] para las Filipinas, que el retorno de ello viene empleado en mercaderías de aquellas provincias que hinchan la Nueva España de manera que es causa que cese la contratación y ventas de las mercaderías de España, demás de la falta de aquellos dos millones de plata que, si vinieran a España, causaran muy grandes derechos a la Hacienda Real en el empleo y cargazón de frutos de la tierra y con esta plata fuera fácil despachar cada un año flota.

Para los consulares, los consiliarios y los grandes cargadores a Indias, la mejor solución para aquel problema revestía pocos misterios: «conviene a el servicio de Su Majestad que cese la dicha contratación de Nueva España a las Filipinas y se haga desde España». ${ }^{42}$

Dicha afirmación constituía algo así como un programa de máximos, que no intentaba justificarse con argumentos vinculados a intereses propios, sino a través de referencias a un clima de competitividad entre estados europeos que afectaba a la monarquía hispánica en general y a los intereses de la corona muy en particular. Pese a ello, los cargadores a Indias también tenían un programa de mínimos orientado a sus necesidades más urgentes y perentorias. Pretendían que se les concediera un permiso para retrasar los pagos a sus proveedores en Sevilla, a cambio de un incremento razonable en el porcentaje de los intereses. Y no se lo solicitaron a sus acreedores, sino a

42 AGI, Consulados, 1, 224v-226v. 
las instituciones para que se lo impusiesen, otorgándoles a ellos un evidente trato de favor. Procuraban obtener lo que se conocía como una cédula de espera, que a quien hacía esperar era al acreedor, no al deudor, evidentemente. Los sevillanos sabían que con la monarquía era mejor negociar puntos concretos y realistas, antes que discutir sobre grandes diseños imperiales en los que la corona era normalmente reacia a introducir cambios significativos inspirados por aspiraciones sectoriales localizadas. ${ }^{43}$

La cédula de espera fue concedida por Felipe III, ${ }^{44}$ tal como solicitó el consulado. Pero ni él ni Felipe IV vetaron el comercio entre Filipinas y México, tal como proponía Levanto. Lo regularon, pero no lo impidieron, a despecho de los deseos de las comunidades mercantiles de Andalucía. Dos discusiones sobre un mismo problema, sostenidas en coyunturas próximas en el tiempo, cosecharon resultados completamente diferentes. Se rechazaron las modificaciones estructurales y se aprobaron eventualmente las coyunturales, de menor alcance y dimensión, más sencillas de aplicar también. El hecho permite algunas reflexiones específicas, en las que no se entrará aquí. Lo que interesa es comprobar cómo un mismo problema comercial de la carrera de Indias podía tratarse tanto desde la perspectiva individual de un arbitrista como desde la perspectiva institucional. Y, en último extremo, cómo la vía institucional cosechaba un éxito mayor. Solo es un caso concreto, pero, puesto en relación con los demás datos y argumentos que se han señalado en las anteriores páginas, permite reforzar la idea de que el sistema institucional controló la vida política de la carrera durante las primeras décadas del siglo XVII. Ese dominio desincentivó el desarrollo de una cultura arbitrista propia del comercio colonial. Debe tenerse por un ejemplo de la fortaleza institucional del modelo imperial hispano, tal como ha destacado Antonio M. Bernal. ${ }^{45}$

\section{La fase gaditana: represión}

Evidentemente, la desincentivación del arbitrismo individual en la Sevilla indiana se debe al razonable entendimiento entre la comunidad de mercaderes y la estructura institucional. Sin embargo, cuando Cádiz y sus mercaderes ganaron protagonismo en la carrera durante la segunda mitad

43 Díaz Blanco, 2012a, cap. 2.

44 AGI, IG, 754, Real Cédula, Madrid, 13 de diciembre de 1610.

45 Bernal, 2005, 177 y ss. 
del siglo XVII, la proximidad respecto a la estructura institucional sevillana ya no pudo ser la misma. A principios del siglo XVII, el conglomerado Consulado-Casa-Consejo podía representar relativamente bien a la sociedad mercantil, o al menos a sus elementos más dinámicos. En las últimas décadas del siglo XVII, ya no era así. Más aun, la sociedad empresarial gaditana, o una parte de ella, aspiraba a modificar la estructura institucional. Así, durante la segunda mitad del siglo XVII, la contradicción entre el modelo institucionalizado de la carrera y la pulsión individualista del arbitrismo se convirtió casi en una confrontación. No es que las instituciones desincentivasen las iniciativas aisladas, es que las iniciativas aisladas entraron en competencia directa con la supervivencia del stablishment.

Seguramente, ninguna trayectoria resume mejor este proceso que la de Juan Cano, a quien se conoce fundamentalmente por el estudio que le dedicó Manuel Herrero Sánchez. ${ }^{46}$ Tras largos años de viajes y reflexión, Cano presentó en la corte de Madrid el primer fruto de su odisea personal en una serie de memoriales y opúsculos impresos entre octubre de 1674 y abril de 1675. La mayoría iba dedicada a la regente doña Mariana de Austria, que remitió su estudio al Consejo de Indias a través de cuatro decretos. ${ }^{47}$ El primer memorial fue un adelanto de su pensamiento que comenzaba con una exaltación de la monarquía, la mayor de todas las del mundo, «pues que domina en sus quatro partes», nacida de sus cenizas después de «restaurarse del bárbaro poder» del Islam y «ponerse en estado de conquistar todo el mundo viejo, como hizo el nueuo». Pese a ello, la «natural generosidad, y demasiada confiança, en las [riquezas] futuras que quedan en Indias» habían provocado una situación de emergencia frente a la que había que reaccionar. Cano detectaba doce perjuizios, para cuya solución proponía otros tantos estatutos, término que en su lenguaje personal parece sustituir a los arbitrios. ${ }^{48}$ El 12 de enero de 1675, Cano presentó el librito en el que desarrollaba sus ideas, titulado Reformacion moral, politica y christiana del comercio, en doze estatutos que restauran treinta millones de reales de a ocho de renta cada año a la Monarquia española. ${ }^{49}$

46 Herrero Sánchez, 1994.

47 AGI, IG, 638, Mariana de Austria al conde de Medellín, Madrid, 21 de noviembre de 1674, 21 de enero, 6 de marzo y 5 de mayo de 1675.

48 AGI, IG, 638, Informacion de los perjuizios que causa el comercio, ò modo como se haze, con los medios para su reformación, en seruicio de Vuestra Magestad, beneficio de toda la Monarquia, sin perjuizio del mismo, s.f. [Madrid, octubre de 1674].

49 BN, $1 / 43493$. 
Después entregó otros tres que lo complementaban ${ }^{50} \mathrm{y}$ «demás escritos conducientes à este fin». ${ }^{51}$ Aquel año de 1675 los consejeros de Indias se enfrentaron a una copiosa cantidad de papel, portadora de los desvelos y ocurrencias de aquel borgoñón entusiasta.

La tesis central de Cano afirmaba que el comercio era la actividad que ensalzaba y hundía a los estados..$^{52}$ Por tanto, su restauración traería consigo la restauración de la monarquía hispánica. Sin embargo, los españoles estaban «tan lexos de la practica, y perfecta conocencia desta tan mercenaria profession, quanto sabios, y expertos [son] en las políticas de materia de Estado, y gouierno»..$^{33}$ Era el comercio internacional lo que provocaba la decadencia española y el auge de los enemigos. Pero aquella situación podía revertirse y los medios principales para conseguirlo serían los siguientes:

- Centralización en la ciudad de Cádiz de toda la actividad institucional, empresarial y administrativa relacionada con el comercio de las Indias, vinculada a transformaciones en el sector secundario implantadas a lo largo y ancho de la geografía española.

- Establecimiento de una «gran Compañia general» que aglutinase a todos los mercaderes.

- Adecuación de las instituciones fiscales de la monarquía para que lograsen extraer con eficiencia recursos tributarios más adecuados.

- Fundación de diez fábricas de textiles de seda y cinco de productos de lana, que dotasen al comercio español de una producción propia y evitasen el recurso a las manufacturas extranjeras, con la consiguiente huida de metales preciosos fuera de las fronteras de Castilla.

50 1) Breue informacion de la proposicion tocante a la reformacion del comercio, verificacion de su contenido y manifiesto de la solicitud del autor, Madrid, 1675 (BN, 3/49748-1); 2) Manifiesto de la proposicion tocante a la informacion de la reformacion del comercio y ofrecimiento de su iustificacion, Madrid, 1675 (BN, 3/49748-2); 3) Doze peticiones, o svplicas, a favor de la prosperidad de la Monarqvia española, a las personas principales en ella interessada. Por Ivan Cano, natvral de la Villa de San Claudio, en el Condado de Borgoña, Madrid, 1675 (BN; 2/62504 y AGI, IG, 638). Cano los menciona abreviadamente como «Breue Informacion, Manifiesto, y doze Peticiones à las personas interessadas en la prosperidad de esta Monarquia» y les da la categoría de libros, aunque tuvieran en realidad una extensión muy mediana. El expediente administrativo indica claramente que esos otros tres «libros» fueron entregados en fechas posteriores al 12 de enero.

51 Se trata de los memoriales impresos que se han conservado en AGI, IG, 638 (ver apéndice).

52 Memorial B.1: «el comercio que oy tiene en toda la tierra tanto poder, por ser dispensador de las riquezas que leuanta los menores Estados, y Republicas al mayor trono, en competencia, y declina las mayores Monarquias».

53 Memorial B.1. 
- Dotación a Cádiz de las infraestructuras necesarias para capitalizar el cambio. Era especialmente importante la construcción de un magno edificio llamado de Puerto Franco, que

no solo será casa de Comercio, sino habitación de los que ocuparen los principales cargos de la Corona, como son Generales, Almirantes de las Armadas de su Magestad Virreyes, Capitanes, y Gouernadores, q deueran pasar, asistir, ò habitar en la Ciudad de Cadiz, donde deue ser fabricado, por bastantes razones. Y tambien viuienda, y Tribunales de los Presidentes, Iuezes, y Gouernadores de la gran Compañia General, de la Contratacion, y del dicho Edificio, en la froma contenida en los Estatutos de su reformación. Como tambien será habitacon de los Gouernadores de dicha Ciudad, Alcaides, o Castellanos de la fortaleza. Y lo mas propio de la Real obstentacion del Monarca será, que tendrá su Magestad viuienda capacissima, quando su Real persona quisiera pasar à dicha Ciudad a ver sus aumentos, y la prosperidad de sus Reales Armadas, que se preuiene por la construcción de dicho Edificio.

- Cano cuidó este arbitrio con especial detalle e incluso trazó un bellísimo proyecto del edificio, ${ }^{54}$ acompañado de una detallada descripción de sus partes. ${ }^{55}$ Lo presentó todo directamente al conde de Medellín, a quien adjuntó igualmente un memorial impreso con más razones a favor del proyecto. ${ }^{56}$

En definitiva, Cano apostaba por la destrucción del modelo monopolístico creado en Sevilla a comienzos del siglo XVI e, implícitamente, de algunas de sus principales instituciones, especialmente el consulado. A cambio, proponía la creación de otro modelo diferente, que aceptase el hecho del nuevo protagonismo gaditano e imitase algunas de las novedades técnicas de signo mercantilista introducidas en la economía europea durante las décadas anteriores. España aprovecharía su rico comercio cuando contase con unidades de producción adecuadas y entidades administrativas eficientes que extrajesen recursos fiscales suficientes para la monarquía, ideas no muy diferentes a las expresadas por Sancho de Moncada en 1619. El símbolo del cambio sería la macroestructura levantada en Cádiz, en la

54 AGI, Mapas y Planos, Europa y África, 115.

55 AGI, IG, 638, Descripcion de la traza del edificio, contenido en los escritos presentadosa à los Ministros del Real Consejo de Indias, de mandado de su Magestad. Por Iuan Cano, natural de la Villa de San Claudio, en el Condado de Borgoña, s.f. [Madrid, 1675].

56 AGI, IG, 638, Memorial al excelentissimo señor Conde de Medellin, con la presentación de la planta del Edificio de Puerto Franco, dedicada a su Excelencia, por Iuan Cano, natural de la Villa de San Claudio, en el Condado de Borgoña, s.f. [Madrid, 1675]. De este documento se ha extraído la cita presentada en este punto, que podría continuarse, pues a renglón seguido Cano también ofrece el edificio como espacio de recreo de las reinas de España y pondera sus bajos costes de construcción. 
que coincidirían los ministros de la monarquía, los militares, las autoridades locales y la gran compañía comercial en la que deberían integrarse los mercaderes. Incluso podría acudir allí la corte cuando fuese apetecido. Cano soñaba con traer a Cádiz el poder político de la monarquía y acercarlo al poder económico del comercio, que no podía trasladarse a Madrid. Una nueva edad para el país.

Cano presentó sus últimos escritos el 17 de abril. Después, afirmó, «no le queda en esta empressa que cumplir de sus escritos, solicitud personal, que hazer, ni experiencia de su capacidad, que manifestar». No deseaba permanecer en Madrid, donde el conde de Medellín le había prometido una pronta respuesta a sus arbitrios que no había llegado en el plazo prometido. Solicitó entonces acudir a Cádiz, «cuya familia, y intereses padecen por falta de su presencia» después de tres años de ausencia. Pero no solo quería volver para reencontrarse con sus seres queridos o atender sus asuntos, sino para seguir escribiendo. Escribir, la pasión de su vida y de tantos otros arbitristas. Quería publicar unos «tratados de conservación, y aumento de Indias, que ofrece, que serán diuersos tomos: Y otros tratados Militares de Mar y Tierra, conformes en numero de Soldados, Baxeles, y Galeras». Quería dar a la luz «vn tratado general de todas las materias, tocante à la prosperidad desta Monarquia, Y pertenecientes à todos los Reales Consejos de V. Mag. Con política vniuersal en tiempo de paz, y guerra, sacada de las antiguas, y modernas». La obra sería tan considerable que, «por ser diferentes tomos, con escriuir, y estampar cada dia tres pliegos de papel, será necesario vn año al menos para su consecución». ${ }^{57}$

Pese a la fiebre de la pluma, sus inquietudes no eran solo intelectuales, sino también prácticas. Cano se ofreció voluntario para cruzar el océano y servir a la monarquía como visitador de algún reino en particular o incluso de todos los virreinatos. La exuberancia de su prosa barroca expresó de esta manera los beneficios que podrían deducirse de su servicio a la corona:

Se expone a pasar à Indias de mandado de V.M. para los aumentos de las sacas de oro, y plata, Conservacion de los Indianos, q[ue] beneficia sus minerales, como de esmeraldas, y demàs piedras preciosas, pescas de perlas, Y descubrimiento de Reynos Gentiles circuvezinos, à quien sobra las riquezas, que faltan en nuestra Europa, y falta la luz de la Fè, que sobra en nosotros, Mediante el empadronamiento de sus tributarios, en la forma que tiene dispuesta, y apuntada en sus escritos, con la conquista

57 Memorial B.2. 
final del Reyno de Chile, y otros, deseosos de gozar libres de lo q[ue] sus vecinos oprimidos desemparan: Cuyos escarmientos en cabeça agena, naturalmente impiden la propiedad destas conquistas y la prospera conservación de los Imperio del Nueuo Mundo.

Detrás de todo había una pretensión económica: la concesión de una pensión de 6.000 pesos anuales situados en Cádiz. ${ }^{58}$

Cano se temía una resolución desfavorable sobre sus arbitrios comerciales, ${ }^{59}$ pero es difícil que pudiese prever la reacción que su actividad generó y que tanta amargura habría de causarle. Las autoridades no solo le negaron el permiso para pasar a Cádiz o a Indias, sino que incluso consideraron oportuno retenerlo en una prisión de la corte. Al parecer, nadie quería que escribiese ni opinase tanto sobre materias tan delicadas. Ni que fuese a América a visitar todo el territorio o a inmiscuirse en la terrible Guerra del Arauco. O que se permitiese infravalorar públicamente y por escrito a los ministros de Su Majestad, actitud que cultivó con excesiva imprudencia. Cano fue encarcelado por razón de estado. Paralelamente, los arbitrios sobre comercio que había presentado el año anterior conocieron el más rotundo desprecio. El 13 de mayo el conde de Medellín se reunió con la plana mayor de sus consejeros para reflexionar sobre las ideas de aquel arbitrista impertinente. ${ }^{60} \mathrm{El}$ parecer fue devastador, despectivo y quedó reflejado sin ningún pudor en el texto de la consulta definitiva que se elevó a doña Mariana, fechada el día 28. Merece la pena leerlo directamente para comprobar como los ministros españoles despreciaban todo aquello que no les convenía y no encajaba dentro de sus esquemas.

Y hauiendosse visto en el Consejo (como V. Mag[esta]d. lo manda) todas las proposiçiones referidas y conferídosse sobre ellas con particular cuidado y atencion, se reconoce el buen çelo con que Juan Cano se a aplicado a discurrir en esta materia por lo que ha visto practicar en otros Reynos y Prouinçias, pero como las de España se gouiernan con diferentes leyes y ordenanzas, se considera por inpracticable e impo-

58 Idem.

59 Lo temía ya, al menos, desde la redacción del memorial B.7, que servía de presentación a uno de los libros complementarios de la Reforma moral, política y cristiana del comercio.

60 AGI, IG, 638, Parecer del Consejo, Madrid, 13 de mayo de 1675. En el sobrescrito del decreto de 5 de mayo, citado supra. Tomaron parte en las deliberaciones: el presidente conde de Medellín, Tomás de Valdés, Antonio de Castro, Juan de Santelices, José Ponce, Juan del Corral, Antonio Ronquillo y Bernabé Ochoa de Chinchetru, cuyos nombres aparecieron todos al margen de la consulta final, aunque Santelices no la rubricó. Después, Valdés se encargó de reproducir al secretario Francisco Fernández de Madrigal el texto que reflejase adecuadamente el parecer del Consejo y éste se lo remitió al conde de Medellín para preparar la redacción definitiva de la consulta: Fernández de Madrigal a Medellín, Madrid, 24 de mayo de 1675, que respondió al día siguiente. 
sible de executarse todo lo que propone, assí en lo espiritual como en lo temporal, pues ello mismo está manifestando que los presupuestos que hace son fantásticos y sin ningún fundamento ni sustancia y quando fuera posible que se pudiessen practicar los estatutos que forma para el Comerçio, es mui digno de reparo, porque con esta nouedad se alteraría todo el orden y gouierno estableçido en quanto a la forma del Comerçio con leyes tan justas y proporçionadas a la calidad y naturaleça de cada Prouinçia, de que resultaría la turbaçión que se deja considerar y siendo esta materia tan vniuersal y que toca el tratar de ella por lo que mira a estos Reynos a otros Consejos, Deue representar éste por lo que pertenece al Comerçio de las Indias que de ninguna manera es practicable ninguno de los medios que propone Juan Cano, y assí pareze al Consejo que se deuen menospreciar sin dar oýdos a semejante nouedad, sino que totalmente se excluya por lo que mira a las Indias.

Después de semejante exabrupto, a los consejeros no se les olvidó referir su voluntad de someterse a la resolución real. Pero la respuesta de Mariana de Austria, una mujer que se encontraba ya en el ocaso de su poder, ${ }^{61}$ difícilmente habría podido ser otra diferente a la que garabateó con cierta indiferencia sobre el sobrescrito de la consulta: «confórmome con lo que pareze». ${ }^{62}$

El que no podía conformarse era Cano. Habría sido difícil que aceptase pasivamente aquella decisión y que se resignase a permanecer en prisión como pago a tantos desvelos, tantos pensamientos y tantas horas volcando sus obsesiones sobre montones de papel. Intentó cambiar su suerte con escritos en los que se reafirmaba en sus ideas y en los que atacaba abiertamente a los consejeros de Indias que lo habían despreciado. ${ }^{63}$ Pero no tuvo ningún éxito ${ }^{64}$ y terminó su aventura personal tachado de arbitrista con pensamientos «fantásticos y sin fundamento». Despreciado y denostado. Quiso enderezar la monarquía y acabó odiado por sus ministros. La contemplación de su triste experiencia personal invita a replantearse el tópico literario sobre el arbitrista, que tal vez no sea tan divertido como puede parecer al principio. El juicio desdeñoso de los consejeros de Indias recuerda la prosa de Cervantes, Saavedra Fajardo o Gracián y la dramaturgia de Tirso, Alarcón o Moreto. Remite, sobre todo, a aquel arbitrista que pasea su ridiculez por las páginas de La niña de los embustes Teresa de Manzanares

61 Oliván Santaliestra, 2006.

62 AGI, IG, 638, Consulta del Consejo de Indias, Madrid, 28 de mayo de 1675. La parte expositiva de la consulta se nutre, fundamentalmente, de los memoriales A.1 y B.2.

63 AGI, IG, 638, Cano a Medellín, «de la cárcel», s.f. [Madrid, julio de 1675] y memorial B.8.

64 AGI, IG, 638, Parecer del Consejo de Indias, Madrid, 29 de julio de 1675; Parecer del fiscal Pedro Fernández Miñano, Madrid, 30 de julio de 1675; Parecer del Consejo de Indias, Madrid, 1 de agosto de 1675. Todos en el sobrescrito del memorial de Cano. 
(1632), de Alonso de Castillo Solórzano: «habiendo este hombre presentado sus memoriales en el Consejo y comunicado con los ministros de él su arbitrio, viendo ser sin pies ni cabeza, no solo no le admitieron, más, por eximirlo de sus cansancios y necias máquinas, le mandaron que dentro de ocho días saliese desterrado de la corte». ${ }^{65}$ Cuarenta años después, Juan Cano se vio en la misma situación que el personaje novelesco.

Sin embargo, Cano no era un fantástico sin fundamento, ni sus escritos eran «sin pies ni cabeza». Basta leerlos para darse cuenta. Del arbitrista de Castillo Solórzano no pueden conocerse ni sus arbitrios ni la opinión que guardaba de los consejeros que se burlaron de él. El novelista no quiso dar esas claves al lector. Pero sí se conoce el perfil que se formó Cano sobre los consejeros de Indias; los deploraba tanto como ellos a él y los consideraba ineficaces, ignorantes e incapaces de tratar las muchas materias que tenían entre manos. Lógicamente, eso tampoco era cierto. Los consejeros de Indias, con todos sus defectos personales y corporativos, formaban parte de la élite de la política española y contaban con una formación personal óptima, según los esquemas de la época. Era el odio mutuo lo que generaba las críticas recíprocas y ese odio procedía de la diversidad de opiniones y de la incapacidad de dialogar.

Los proyectos de Cano, y esto es esencial para garantizar la representatividad de su experiencia, no fueron hechos aislados. A lo largo de la segunda mitad del siglo XVII pueden encontrarse otras propuestas destinadas a modificar estructuralmente la carrera de Indias, dirigidas a crear compañías comerciales privilegiadas que empezasen a protagonizar la vida de la economía atlántica. Ninguna salió adelante, al igual que las ideas de Cano. Manuel Bustos Rodríguez ha estudiado el intento de crear una compañía general de comercio de los Países Bajos, que no llegó a nada. ${ }^{66}$ Otro caso fue el proyecto de levantar una compañía general de comercio en Castilla, que se debatió al menos en 1685 en una junta formada por miembros de diferentes consejos. ${ }^{67}$ Las ideas económicas respecto al comercio colonial adquirieron riqueza a lo largo de la segunda mitad del siglo XVII en el pensamiento de Manuel de Lira, Miguel Álvarez Ossorio y Redín o fray Juan de Castro, ${ }^{68}$ aunque con escasas repercusiones prácticas. El fraca-

65 Vilar, 1973, 89.

66 Bustos Rodríguez, 1999.

67 AGI, IG, 644, Decreto de Carlos II, Madrid, 5 de marzo de 1685.

68 Muñoz Pérez, 1957. Entre estos autores, ninguno de los cuales era cargador a Indias, Lira ofrece otro ejemplo de discrepancia ideológica y alejamiento personal con la cúpula política de la monarquía: Bernal, 2005, 196-197. 
so de estas ideas y de quienes las defendían se debe al choque que se producía inevitablemente contra los privilegios y las prerrogativas de las instituciones tradicionales de la carrera. Nada cambió mientras el gobierno de Carlos II mantuvo la misma convicción: «conviene a mi servicio conservar en reputación y caudal al Consulado y comercio de Sevilla» ${ }^{69}$ Esos privilegios eran la contrapartida a las grandes sumas de dinero que el consulado puso a disposición de la monarquía durante las últimas décadas del siglo, entre 1680 y 1700, aproximadamente. Las ansias de renovación sucumbían ante las tendencias conservadoras del poder establecido.

\section{De la desincentivación a la represión, de Sevilla a Cádiz: una oportunidad para reflexionar sobre el arbitrismo}

José Luis Sureda Carrión, al estudiar a los que denominaba los «economistas del siglo XVII», advirtió que «el punto de vista común en el tratamiento de los problemas económicos por los autores que estudiamos lo daba la creación política del Renacimiento: el Estado». ${ }^{70}$ Esta afirmación clásica ha encontrado una confirmación reciente en la innovadora visión ofrecida por Luis Perdices de Blas y John Reeder, que han integrado plenamente el pensamiento arbitrista dentro de las corrientes mercantilistas europeas. El arbitrismo habría sido la principal rama española del mercantilismo que, según los autores, no debe definirse como un «cuerpo sistemático de ideas», sino como un «conjunto de propuestas de política económica desarrollista cuyo fin último es la consolidación del poder del Estado por medio del fomento de la economía». ${ }^{71}$ La afirmación admite pocas dudas, habida cuenta de la omnipresente preocupación de los arbitristas del XVII por salvar y desempeñar el erario regio. Resulta indudable: el arbitrismo fue un tipo de pensamiento funcional, en el que la especulación careció de una relevancia per se. Por tanto, se hace necesario comprender las vinculaciones reales entre los arbitristas y las instituciones a las que pretendían fortalecer. Dicho de otro modo, el análisis del arbitrismo no puede ser completo si solo se atiene a los textos y se olvida los contextos.

69 AGI, IG, 648, Decreto al marqués de los Vélez, Madrid, El Escorial, 27 de octubre de 1692.

70 Sureda Carrión, 1949, 105. estudio).

71 Perdices de Blas, 1996 y Perdices de Blas y Reeder, 1998 (la cita proviene de este segundo 
Los resultados de este enfoque entusiasman cuando se toma como referencia a los grandes arbitristas, que escribían con un cierto conocimiento de causa y un demostrado impacto institucional. Esos son indispensables para entender determinados momentos de la vida política castellana. El problema es que el método se ha puesto mucho menos en práctica para estudiar a pensadores de menos fortuna. Dicho de otro modo, la investigación se ha centrado preferentemente en los ejemplos de colaboración eficaz entre la monarquía y los arbitristas, en coherencia consciente o inconsciente con la definición de estos como constructores del estado moderno y la economía productiva. Los que no colaboraron con la monarquía han seguido siendo los ingenuos e incompetentes de los que nada interesante cabe decir. Hasta cierto punto, se ha asumido acríticamente con cierta frecuencia la cultura anti-arbitrista del siglo XVII. A pesar de las diversas trampas que pueden existir detrás de ella, sobre las que nos han advertido Vilar y Dubet.

Sin dejar de admitir que muchos arbitristas pudieran tener una preparación teórica deficiente, no puede admitirse tan simplemente la ecuación de que el buen arbitrista es el arbitrista que fue escuchado por las instituciones. El arbitrista escuchado por las instituciones fue aquel que portaba un mensaje útil y aceptable para el poder político, incluso aunque ese mensaje no contuviera más que ideas comunes y presentara una elaboración técnica mediocre. Del mismo modo, no puede tenerse por cierto que el arbitrista que fuera despreciado por las instituciones fuese necesariamente el mal arbitrista, el chiflado de la literatura satírica aurisecular. Podría serlo en algunas ocasiones, pero otras veces sería aquel que expresaba ideas incómodas para los poderosos, el que propusiese opciones de reforma que amenazaban los privilegios de gente más influyente que él. Eso es algo que no debe olvidarse. Porque, para el historiador, el éxito político es interesante, pero también lo es el fracaso. Es muy significativo el estudio de los pensadores que encontraron acogida entre los políticos de su tiempo, pero también lo es observar cómo otros fueron desanimados y rechazados. Recordar su derrota y la condena social que sufrieron ayuda a no caer en el espejismo, sin duda desacertado, de una colaboración sistemática entre el desarrollo institucional y el mercantilismo arbitrista.

Mientras nuevos descubrimientos no obliguen a rectificar los patrones de análisis, el conjunto de datos que aquí se ha expuesto parece avalar la hipótesis presentada respecto a la existencia de una relación contradictoria entre las instituciones de la carrera y el pensamiento arbitrista. Y respecto 
a su cronología también. Durante las primeras décadas del siglo, los cargadores a Indias se enfrentaron a una considerable batería de problemas, pero tal circunstancia no fomentó el progreso de la cultura arbitrista entre ellos. La respuesta es sencilla: no la necesitaban, porque tenían a su disposición un aparato institucional que los representaba correctamente y trataba con rigor y profesionalidad los asuntos que marcaban la actualidad de entonces. Sin embargo, la evolución del siglo cambió notablemente esta situación. Conforme Cádiz empezó a acaparar mayores cuotas de comercio, creció en ella una comunidad mercantil cuyos intereses ya no estaban tan bien representados por la estructura institucional de la carrera. Más bien, sus intereses eran contrarios a los de la estructura. Así, empezó a surgir un pensamiento arbitrista en Cádiz (o relacionado con Cádiz) que pretendía enfrentarse a las instituciones del eje Madrid-Sevilla, aunque con poco éxito. Como era de esperar, tales instituciones frenaron su avance.

Estos hechos concretos y los que la historiografía ha puesto de relieve en otras ocasiones permiten distinguir varias modalidades de interacción entre el arbitrismo y las instituciones. Todas tienen relevancia para la investigación y ninguna es ajena a cualquier corriente de pensamiento crítico que surge en un espacio donde existe un poder establecido:

- Colaboración arbitrismo-instituciones. Se trata de la posibilidad más contemplada por los historiadores, aquella sin la cual pudiera parecer que no hay materia digna de interés para la investigación. Se produce en varios supuestos: cuando un arbitrista escribe por cuenta propia, pero su mensaje es bien recibido por alguna instancia de poder y lo aprovecha, o cuando son las propias instituciones quienes solicitan el auxilio de un pensador cuyas opiniones pueden resultar de interés.

- Desincentivación del arbitrismo por las instituciones. No se registra un conflicto abierto entre los memorialistas y el poder establecido. Sencillamente, los órganos de gobierno funcionan correctamente y los súbditos representados por ellos no sienten la necesidad de emprender iniciativas propias y presentar escritos individuales con soluciones.

- Rechazo, ignorancia o represión del arbitrismo por las instituciones. Puede justificarse a veces por la conocida ausencia de valor intelectual en los arbitrios de muchos pensadores, pero en otros casos tiene su origen en un conflicto de intereses, sea de la naturaleza que sea. No se escucha al arbitrista porque no conviene o no convence a alguien con poder. Aunque su pensamiento sea brillante o digno de discusión. 
Quienes se encontraban en esta última situación se han visto opacados en el tiempo, confundidos con el tópico literario, que parece tener una dimensión poco jovial que suele pasar desapercibida. Terminaron formando parte del nutrido repertorio de hombres que, a lo largo y ancho de la historia de España, han pensado y sentido de forma diferente a sus contemporáneos. Sobre todo - detalle fatal- a los contemporáneos con auténtica influencia y poder. La consecuencia que sufrieron fue el rechazo, el desprestigio y la ignorancia. En la actualidad se realiza un esfuerzo historiográfico importante por dejar de presentar a los arbitristas como marginados sociales; es decir, terminar con la visión simplista del proyectismo que ha producido el tópico literario. Pero ese esfuerzo reivindicador puede incurrir en el error de olvidar a los que fueron despreciados bajo el pretexto de la extravagancia y centrarse solo en aquellos otros que obtuvieron un cierto reconocimiento social. Cellorigo, Moncada o Valle de la Cerda jamás fueron tenidos por inventores de disparates, porque evidentemente no lo eran. Personajes como Juan Cano sí sufrieron ese estigma, a pesar de que tampoco su pensamiento pueda catalogarse de desvarío. El tópico literario no fue solo literario; para algunos arbitristas, significó una realidad tangible. Sí, hubo marginados entre ellos y explicar su situación requiere contemplar el conflictivo binomio autoritarismo institucional-subjetivismo individual. 



\section{Apéndice}

Se enumeran aquí todos los opúsculos y memoriales impresos de Juan Cano que han llegado hasta la actualidad, conservados en la Biblioteca Nacional de España y en el Archivo General de Indias. Las referencias cronológicas en los memoriales sin título son muy escasas, generalmente no hay fecha, pero todos pueden datarse entre finales de 1674 y principios de 1675, preferentemente entre enero y abril de 1675. Los decretos de Mariana de Austria en los que se han conservado algunos ejemplares pueden ayudar a fecharlos con una precisión algo mayor, pero sin llegar a resolver el problema. Se citan aquí según su orden de aparición en el expediente administrativo, tal y como se conserva actualmente, que no coincide con el orden cronológico de redacción, y a través de sus primeras palabras.

\section{A) Escritos con título}

1. Informacion de los perjuizios que causa el comercio, ò modo como se haze, con los medios para su reformación, en seruicio de Vuestra Magestad, beneficio de toda la Monarquia, sin perjuizio del mismo (AGI, IG, 638, en el decreto de 21 de noviembre de 1674).

2. Reforma moral, política y cristiana del comercio que restaura 30 millones de reales de a ocho de renta a la monarquía española (BN, 1/43493).

3. Breue informacion de la proposicion tocante a la reformacion del comercio, verificacion de su contenido y manifiesto de la solicitud del autor, Madrid, 1675 (BN, 3/49748-1).

4. Manifiesto de la proposicion tocante a la informacion de la reformacion del comercio y ofrecimiento de su iustificacion, Madrid, 1675 (BN, 3/49748-2).

5. Doze peticiones, o svplicas, a favor de la prosperidad de la Monarqvia española, a las personas principales en ella interessada. Por Ivan Cano, natvral de la Villa de San Claudio, en el Condado de Borgoña, Madrid, 1675 (BN, 2/62504 y AGI, IG, 638, dentro del decreto de 5 de mayo de 1675, aunque posiblemente ésa no fue su posición original).

6. Descripcion de la traza del edificio, contenido en los escritos presentados à los Ministros del Real Consejo de Indias, de mandado de su Magestad. Por Iuan Cano, natural de la Villa de San Claudio, en el Condado de Borgoña (AGI, IG, 638, dentro del decreto de 5 de mayo de 1675. Hay otro ejemplar suelto).

7. Memorial al excelentissimo señor Conde de Medellin, con la presentación de la planta del Edificio de Puerto Franco, dedicada a su Excelencia, por Iuan 
Cano, natural de la Villa de San Claudio, en el Condado de Borgoña (AGI, IG, 638, dentro del decreto de 5 de mayo de 1675).

\section{B) Memoriales sin título (AGI, IG, 638)}

1. Señora. Si el zelo, por ardiente, me quitò la ocasion... (en el decreto de 21 de noviembre de 1674, junto con el impreso A.1).

2. Señora. Ivan Cano, Vassallo de V. Mag. humilde... (por duplicado y dentro de un breve billete que lo resume).

3. Señora. Aviendo determinado manifestar à las sagradas plantas... (por duplicado. Este ejemplar acompañaba a los impresos A.4 y A.5, relativos al edificio de Puerto Franco en Cádiz y se entregó al Consejo con el memorial de 5 de mayo de 1675).

4. Señora. En perseverancia del Real servicio de V.R.C...

5. Señora. Ivan Cano, de Nacion Borgoñon, vassallo...

6. Señora. Ivan Cano, Vassallo de V.S.C.R. Magestad... (circuló junto a los memoriales B. 4 y B.5 y todos juntos se enviaron al Consejo a través del decreto de 21 de enero de 1675).

7. Señora. Presentè al Confessor de V. Magestad... (se entregó al Consejo con el decreto de 6 de marzo de 1675, junto a un «libro» que no se identifica).

8. Señora. Por muchas razones S.R. Catolica Magestad deuo... (al final del expediente, junto a una carta de Cano a Medellín, fechable en julio de 1675).

\section{Bibliografía}

Alfonso Mola, Marina y Martínez Shaw, Carlos (coords.): El galeón de Manila. Catálogo, Madrid, Gobierno de España - Fundación Focus, 2000.

Alloza Aparicio, Ángel: «Diseño e implementación de una política mercantilista en el reinado de Felipe IV», en García-Hurtado, Manuel; González Lopo, Domingo y Martínez Rodríguez, Enrique (eds.), El mar en los siglos modernos, Santiago de Compostela, Xunta de Galicia, 2009, 2, 53-66.

Almarza, Sara: Pensamiento crítico hispanoamericano: arbitristas del siglo XVIII, Madrid, Pliegos, 1990.

Archivo General de Indias: Inventario de la sección V Indiferente General, Sevilla, mecanografiado, s.f.

Bernabéu Albert, Salvador (coord.): La nao de China: 1565-1815. Navegación, comercio e intercambios culturales, Sevilla, Universidad, 2014.

Bernabéu Albert, Salvador y Martínez Shaw, Carlos (eds.): Un océano de seda y plata: el universo económico del Galeón de Manila, Sevilla, CSIC, 2013. 
Bernal, Antonio Miguel: «La Casa de la Contratación de Indias: del monopolio a la negociación mercantil privada (siglo XVI)», en Vila, Acosta y González, 2003, 129-160.

Bernal, Antonio Miguel: España, proyecto inacabado. Costes/beneficios del Imperio, Madrid, Marcial Pons, 2007.

Collado Villalta, Pedro: «El Consulado de Sevilla: por un mayor protagonismo en la carrera de Indias, 1591-1608», en Andalucía y América en el siglo XVI, Sevilla, EEHA-CSIC, 1983, 275-305.

Colmeiro Penido, Manuel: Biblioteca de los economistas españoles de los siglos XVI, XVII y XVIII [1861], Luis Perdices de Blas y John Reeder (eds.), Madrid, Real Academia de Ciencias Morales - Instituto de Estudios FiscalesFundación ICO, 2005.

Correa Calderón, Evaristo: Registro de arbitristas, economistas y reformadores españoles (1500-1936). Catálogo de impresos y manuscritos, Madrid, FUE, 1981.

Crespo Solana, Ana: La Casa de Contratación y la Intendencia General de la Marina en Cádiz, Cádiz, Universidad, 1996.

Díaz Blanco, José Manuel: «La monarquía de Felipe III: un "cuerpo místico” y desequilibrado», en Actas de la IX Reunión Científica de la FEHM, Málaga, FEHM, 2009a, 2, 409-424.

Díaz Blanco, José Manuel: «La extinción de las naturalezas en la carrera de Indias (un episodio de la caída del conde duque de Olivares)», en Estudios de Historia Moderna en homenaje al profesor Antonio García-Baquero, Sevilla, Universidad, 2009b, 321-336.

Díaz Blanco, José Manuel: Así trocaste tu gloria. Guerra y comercio colonial en la España del siglo XVII, Madrid, IUHS-Marcial Pons, 2012a.

Díaz Blanco, José Manuel: «América en el pensamiento arbitrista: propuestas proteccionistas para la crisis castellana del siglo XVII», en Iñesta Mena, Félix y Mateos Ascacíbar, Francisco J. (coords.), España: Nación y Constitución y otros estudios sobre Extremadura, Llerena, Sociedad Extrema de Historia, 2012b, 77-90.

Domínguez Ortiz, Antonio: Los extranjeros en la vida española durante el siglo XVII y otros estudios, Sevilla, Diputación, 1996.

Dubet, Anne: «L'arbitrisme: un concept d historien?», Cahiers du Centre de Recherches Historiques, 24, 2000, edición digital: http://ccrh.revues.org/ 2062.

Dubet, Anne: Hacienda, arbitrismo y negociación política. Los proyectos de erarios públicos y montes de piedad en los siglos XVI y XVII, Valladolid, Universidad, 2003.

Elliott, John H.: «Introspección colectiva y decadencia en España a principios del siglo XVII», en Elliott, John (ed.), Poder y sociedad en la España de los Austrias, Barcelona, Crítica, 1982, 198-223. 
Elliott, John H.: El conde duque de Olivares. El político en una época de decadencia, Barcelona, Crítica, 1990.

Falcón Ramírez, Javier: Clases, estamentos y razas. España e Indias a través del pensamiento arbitrista del marqués de Varinas, Madrid, CSIC, 1988.

Fernández de Navarrete, Pedro: Conservación de monarquías y discursos políticos [1626], Madrid, Instituto de Estudios Fiscales, 1982.

Feros Carrasco, Antonio: El duque de Lerma. Realeza y privanza en la España de Felipe III, Madrid, Marcial Pons, 2002.

García Guerra, Elena M: Moneda y arbitrios: consideraciones del siglo XVII, Madrid, CSIC, 2003.

García Fuentes, Lutgardo: Los peruleros y el comercio de Sevilla con las Indias, 1580-1630, Sevilla, Universidad, 1997.

García Pérez, Rafael: El Consejo de Indias durante los reinados de Carlos III y Carlos IV, Pamplona, Universidad, 1998.

González de Cellorigo, Martín: Memorial de la política necesaria y útil restauración a la república de España y del desempeño universal de estos reinos [1600], Madrid, Instituto de Estudios Fiscales, 1991.

Heredia Herrera, Antonia: Inventario de los fondos de Consulados (sección XII) del Archivo General de Indias, Madrid, Ministerio de Cultura, 1979.

Heredia Herrera, Antonia: «Los dirigentes oficiales del Consulado de Cargadores a Indias», en Torres, Bibiano y Hernández, José J. (coords.), Andalucía y América en el siglo XVII. Actas de las III Jornadas de Andalucía y América, Sevilla, CSIC, 1985, 216-236.

Herrero Sánchez, Manuel: «Cádiz y la reorganización del comercio indiano en el proyecto mercantilista de Juan Cano (1675)», en Andalucía y América. Actas del II Congreso de Historia de Andalucía, Córdoba, Junta de Andalucía, 1994, 171-178.

Herzog, Tamar: «El arbitrismo y América», en Sabatini y Dubet, 2008, 925-931.

Larraz, José: La época del mercantilismo en Castilla (1500-1700), Madrid, Aguilar, 1963.

Martínez de Mata, Francisco: Memoriales y discursos, Madrid, Moneda y crédito, 1971.

Martínez Millán, José y Visceglia, Maria Antonietta (dirs.): La monarquía de Felipe III: la corte, Madrid, Mapfre, 2008.

Martínez Shaw, Carlos y Alfonso Mola, Marina: La ruta española a China, Ed. El Viso, Madrid, 2007.

Moncada, Sancho de: Restauración política de España [1619], Madrid, Instituto de Estudios Fiscales, 1974.

Muñoz Pérez, José: «El comercio de Indias bajo los Austrias y los tratadistas españoles del siglo XVII», Revista de Indias, XVII-68, Madrid, 1957, 209-221.

Oliván Santaliestra, Laura: Mariana de Austria. Imagen, poder y diplomacia de una reina cortesana, Madrid, Universidad Complutense, 2006. 
Perdices de Blas, Luis y Sánchez Molledo, José María (eds.): Arbitrios sobre la economía aragonesa del siglo XVII, Zaragoza, Universidad, 2007.

Sabatini, Gaetano y Dubet, Anne (coords.): «Arbitristas: acción política y propuesta económica», en Martínez Millán y Visceglia, 2008, III, 867-936.

Schäfer, Ernst: El Consejo Real y Supremo de las Indias: su historia, organización y labor administrativa hasta la terminación de la Casa de Austria [1935], Valladolid, Junta de Castilla y León-Marcial Pons, 2003.

Schurz, William Lytle: El galeón de Manila [1939], Madrid, Ediciones de Cultura Hispánica, 1992.

Sureda Carrión, José Luis: La Hacienda castellana y los economistas del siglo XVII, Madrid, CSIC, 1949.

Tariego, Antonio de (dir.): Inventario analítico de los papeles que vinieron de la Contratación de Sevilla a este Archivo General de Indias. Tomo IV y último, Sevilla, manuscrito, 1801.

Vila Vilar, Enriqueta: «El poder del Consulado sevillano y los hombres del comercio en el siglo XVII: una aproximación», en Vila Vilar, Enriqueta y Kuethe, Allan (coords.): Relaciones de poder y comercio colonial: nuevas perspectivas, Sevilla, CSIC - Texas Tech University, 1999, 3-34.

Vila Vilar, Enriqueta; Acosta, Antonio y González, Adolfo (coords.): La Casa de la Contratación y la navegación entre España y las Indias, Sevilla, CSIC, 2003.

Vilar, Jean: Literatura y economía. La figura satírica del arbitrista en el Siglo de Oro, Madrid, Revista de Occidente, 1973. 